\title{
A ATUAÇÃO DA FISIOTERAPIA NA FASE PRECOCE EM PACIENTES COM A DOENÇA DE ALZHEIMER: UMA REVISÃO BIBLIOGRÁFICA
}

\author{
Ana Julia Oliveira Moreira ${ }^{1}$
}

RESUMO: Esta pesquisa se justifica em analisar como a fisioterapia pode atuar de forma positiva em pacientes com Alzheimer, para a sua plena recuperação, baseando-se nas ideias de diferentes autores e protocolos da área. Realiza-se, então, uma pesquisa bibliográfica, através da técnica de revisão de literatura, sendo utilizados artigos relacionados ao tema em questão, em língua portuguesa, e consulta nas bases de dados PubMed-Medline da United States National Library of Medicine, National Institutes of Health e Scielo - Scientific Electronic Library Online e Google Acadêmico, separando os artigos de 2010 até 2019. Sendo que os descritores/palavras-chave utilizados nesta pesquisa foram: fisioterapia neurológica, atuação do fisioterapeuta na doença de Alzheimer e tratamento precoce. Sobre a doença de Alzheimer, constata-se que esta é a forma mais comum de demência neurodegenerativa, em pessoas de idade, sendo sua causa ainda gera mistério para os pesquisadores da área médica, mas acredita-se que seja geneticamente determinada. A doença estabelecer-se em relação ao processamento de determinadas proteínas do sistema nervoso central, que começam a trabalhar de forma incorreta, assim se faz necessário à atuação do profissional de fisioterapia, pois com ela ocorre uma diminuição dos aspectos motores, principalmente em idosos. Deste modo, este trabalho visa também estudar um tratamento eficaz para o paciente e orientação da família, avaliando este e notando as dificuldades em realizar as atividades cotidianas, avaliando marcha, entre outros pontos e montar um tratamento coerente, que atenda e supra as necessidades do paciente com Alzheimer. Chegou-se à conclusão de que o papel da fisioterapia é essencial para a melhoria da qualidade de vida dos pacientes portadores de Alzheimer.

Palavras-chave: Doença Alzheimer. Fisioterapia. Atuação precoce. Qualidade de vida.

ABSTRACT: This research is justified in analyzing how physiotherapy can act positively in patients with Alzheimer's, for their full recovery, based on the ideas of different authors and protocols in the area. A bibliographic search is then carried out using the literature review technique, using articles related to the topic in question, in Portuguese, and consultation in the PubMed-Medline databases of the United States National Library of Medicine, National Institutes of Health and Scielo - Scientific Electronic Library

\footnotetext{
${ }^{I}$ Graduanda em Fisioterapia pela Faculdade De Rio Verde Curso- UniBRAS.
} 
Online and Academic Google, separating articles from 2010 to 2019. The descriptors/key words used in this research were: neurological physiotherapy, physiotherapist's role in Alzheimer's disease and early treatment. About Alzheimer's disease, it appears that this is the most common form of neurodegenerative dementia in elderly people, and its cause is still a mystery to researchers in the medical field, but it is believed to be genetically determined. The disease is established in relation to the processing of certain proteins in the central nervous system, which start to work incorrectly, thus it is necessary for the physiotherapy professional to act, because with it there is a reduction in motor aspects, especially in the elderly. Thus, this work also aims to study an effective treatment for the patient and guidance for the family, evaluating this and noting the difficulties in performing daily activities, evaluating gait, among other points and putting together a coherent treatment that meets and meets the needs of the patient. Alzheimer's patient. It was concluded that the role of physical therapy is essential for improving the quality of life of patients with Alzheimer's.

Keywords: Alzheimer's disease. Physiotherapy. Early action. Quality of life.

\section{INTRODUÇÃO}

Este artigo se justifica em analisar a doença de Alzheimer sendo que até o presente momento não se obteve respostas de cura. Uma patologia que atinge a parte neurológica do paciente, causando a demência, atinge na fase dos 60 anos acima, porém, pode ocorrer casos de Alzheimer precoce podendo assim atingir faixa etárias diferente. Esta doença possui três fases, evoluindo da falta de memória até em acometimentos neuro motores e respiratórios (BASTOS,2016).

Assim, a fisioterapia sendo praticada na fase inicial demonstra-se bons resultados no retardamento desta patologia. Visando um tratamento eficaz para o paciente e orientação da família, avaliando o mesmo, notando as dificuldades em realizar as atividades cotidianas, avaliando marcha entre outros pontos de avaliações e a partir disto montar um tratamento coerente, que atenda e supra as necessidades do paciente (KOTTKE, 2012).

Com tudo isso, este trabalho tem como tema a atuação da fisioterapia na fase precoce em pacientes com doença de Alzheimer, sendo inserido na área de concentração da Fisioterapia Neurológica, assim, se quer responder ao seguinte questionamento: De que maneira a fisioterapia pode atuar de forma positiva na fase precoce em pacientes com Doença de Alzheimer? Com tudo isso, chega-se hipótese de que a Fisioterapia inserida em um diagnóstico precoce em pacientes com Alzheimer, pode trazer respostas de forma 
positiva ao retardamento da doença. Podendo assim dar uma qualidade de vida e uma certa independência a este paciente por um tempo maior. Preparando e orientando a família para os cuidados diários com o mesmo (MACHADO, 2012).

Portanto, esta pesquisa tem como objetivo geral: analisar a intervenção fisioterapêutica e como ela pode desacelerar a progressão da doença e como objetivos específicos: discorrer sobre a doença de Alzheimer; analisar as fases de progressão da doença; descrever os protocolos fisioterapêuticos utilizado com o paciente e evidenciar a importância da fisioterapia.

\section{REFERENCIAL TEÓRICO}

\section{2.r Alzheimer: Conceito e Incidência da Doença}

Ao se falar sobre a Doença de Alzheimer, constata-se que esta "é a forma mais comum de demência neurodegenerativa, em pessoas de idade, sendo sua causa mistério para os pesquisadores da área médica, mas acredita-se que seja marcada pela genética de cada um" (CAOVILLA, 2ori, p.or).

A doença estabelecer-se, em relação ao processamento de determinadas proteínas do sistema nervoso central, que começam a trabalhar de forma incorreta, assim se faz necessário à atuação do profissional de fisioterapia, pois com ela ocorre uma diminuição dos aspectos motores, principalmente em idosos (BAPTISTA, 20II).

Os sintomas iniciais são: a perda de memória, o comportamento alterado do indivíduo, com mudanças e picos de humor, com o passar do tempo e da doença, estas perdas são cada vez mais progressivas e comprometem até memórias autobiográficas do doente (MACHADO, 2012).

A doença tem quatro Fases: "Pré-demência: os sintomas são muito sutis e muitas vezes atribuídos, de forma equivocada, ao envelhecimento natural ou ao estresse; Estágio Inicial ou leve; Estágio intermediário e Estágio avançado (terminal) (FLECK, 2010, p.IO2).

As alterações comportamentais desde o princípio são muito costumeiras, no decorrer da doença, indivíduos com alzheimer podem ter características depressivas, de agitação e de agressividade, ou até mesmo delírios e alucinações (FREITAS, 2012). 
Também surgem as mudanças motoras, sendo assim são aplicadas sessões de exercícios cinesioterapêutico para promover: o fortalecimento, equilíbrio, aumentando a coordenação motora, flexibilidade, problemas nas funções respiratórias e a mobilidade das pessoas cometidas pela doença (BERNARDO, 2017).

O diagnóstico é feito através de uma análise clínica da pessoa, pelo médico e a exclusão de outras doenças, por meio de análises de sangue e de imagem (tomografia ou ressonância magnética) e avaliação neuropsicológica (expandida ou computadorizada) (GORZONI, 2016). "Não existe ainda um marcador biológico da doença, ou seja, um exame único que o médico possa pedir e ter a segurança total do diagnóstico" (BALLONE, 2012, p.or).

Diante disso, existem medicações atualmente, que estabilizam a doença ou diminuem a velocidade de perda funcional em cerca de cinco anos ou mais, sendo o caso da memantina, que foi descoberta pelo Alois Alzheimer, podendo oferecendo mais tempo com qualidade de vida, ao paciente e aos familiares. Apesar de a doença não ter cura, as medicações, desde que bem administradas, oferecem: conforto, alívio e qualidade de vida para o doente (XIMENES, 2014).

\subsection{Fisiopatologia do Alzheimer}

Em se tratando da fisiopatologia do alzheimer este se caracteriza, do ponto de vista anatomopatológico, pela presença de placas senis, com situações de agravação do cérebro e das funções motoras (BAUER, 2012).

Sobre as ocorrências clínicas estas ligam-se com estruturas conectadas com a cognição e do comportamento (KAUFFMAN, 2019). "Análises funcionais revelam que as modificações comprometem inicialmente circuitos hipocampais" (CORRÊA, 2012 e BASTOS, 2016, p. 89).

Vários mecanismos já foram descritos como envolvidos na causa da doença de alzheimer como fatores: genéticos, epigenéticos, metabólicos, reações inflamatórias, cascata patogênica mitocondrial, estresse oxidativo, proteínas plasmáticas e cerebrais, fator neurotrófico derivado do cérebro deficiências de estrogênio, além de fatores ambientais (CAVALCANTI e ENGELHARDT, 2012, p.22). 
Para os estudiosos da doença muito interesse tem-se voltado para a influência de fatores vasculares, pois a associação do alzheimer com doença cerebrovascular é muito frequente (BAUER, 2012). "O alzheimer provavelmente é causado por interações patogênicas entre muitos fatores e várias comorbidades, em que ainda não se conhece em que medida cada uma contribui para comprometer as funções neuronais." (CAVALCANTI e ENGELHARDT, 2012, p.23).

Assim, os fatores de risco para o alzheimer são: idade, lesão no crânio, mínima escolaridade, doenças como a hipertensão arterial, hiperlipidemia e diabetes, hábitos alimentares e fatores ambientais variados, e que de acordo com a intensidade cada uma dessas causas influencia na fisiopatologia da doença (BERNARDO, 2018).

Constata-se que, a complexidade e as interrelações de fatores fisiopatogênicos do alzheimer mostram que a sua expressão clínica decorre de um conjunto de fatores, quem estão interligados.

\subsection{Principais Disfunções Funcionais do Alzheimer}

"As principais disfunções funcionais do alzheimer ocorrem no sistema nervoso central e comprometem aspectos neurológicos e funcionais dos indivíduos. Trata-se de uma enfermidade neuropsiquiátrica, que causa variações no estado de ânimo e perda considerável de memória" (PEÇANHA; NERI, 2009, p.oI).

O Alzheimer é a doença que mais provoca quadros de demência, e é caracterizada por: "alterações audi-espaciais; alterações de memória; desorientação em relação ao tempo e ao espaço; desorientação do raciocínio; falta de concentração; distúrbio de aprendizado; impossibilidade de realização de tarefas complexas” (BALLONE, 2012, p. I02).

A evolução dos sintomas da Doença de Alzheimer pode ser dividida em 4 estágios com suas disfunções funcionais:

I. Fase pré-demência ou fase leve é caracteriza pela: perda de memória recente; dificuldade para encontrar palavras; desorientação no tempo e no espaço; dificuldade para tomar decisões; perda de iniciativa e de motivação; sinais de depressão; agressividade; redução do interesse por atividades e passatempos (MACHADO, 2012). 
2. "Comprometimento cognitivo leve: esquecimento leve nas atividades diárias; Dificuldade para lembrar de eventos e atividades; problemas para lembrar o nome de pessoas e coisas" (CHARONE, 202I, p. oI).

3. Demência precoce de estágio moderado ou fase moderada é caracterizada pela: mudança de personalidade; perda de insight; perda de habilidades simples como o hábito de escovar os dentes; não reconhecer pessoas em ambientes familiares; dificuldade em ler, falar e entender as coisas com clareza; confusão, agressividade ou desorientação; inconsciente das limitações pessoais; incapacidade ou grande dificuldade de realizar atividades do dia a dia; lembrança de fatos passado; perda da estabilidade sentimental e comportamental; perda da confiança e segurança; alucinações (ver pessoas, ouvir vozes de pessoas que não estão presentes) (PEÇANHA, 2019).

4. "Demência degenerativa ou fase grave é caracterizada pela: maior desorientação e confusão; completa dependência de cuidados; complicações na saúde física devido a imobilidade; incapacidade de registro de dados e muita dificuldade na recuperação de informações antigas como reconhecimento de parentes, amigos, locais conhecidos; dificuldade para alimentar-se associada a prejuízos na deglutição; dificuldade de entender o que se passa à sua volta; dificuldade de orientar-se dentro de casa; morte por infecções ou outras complicações como, por exemplo, doenças respiratórias. Nessa fase, ainda pode haver incontinência urinária e fecal, intensificação de comportamento inadequado e incapacidade de locomoção" (CHARONE, 202I, p. oI).

Outros sintomas provocados pela Doença de Alzheimer: dificuldade para manusear utensílios, vestir-se, e desenvolver atividades que envolvam autocuidado; alucinações visuais (ver o que não existe) ou auditivas (ouvir vozes) podem ocorrer, sendo mais frequentes da metade para o final do dia, quadros paranóicos; alteração do apetite com tendência a comer exageradamente ou ocorrer diminuição drástica da fome; agitação noturna ou insônia com troca do dia pela noite (GORZONI, 2016).

A Doença de Alzheimer é uma disfunção neurodegenerativa que tem como fatores: genéticos, epigenéticos, metabólicos, reações inflamatórias, cascata patogênica mitocondrial, estresse oxidativo, proteínas plasmáticas e cerebrais, fator neurotrófico derivado do cérebro (ALCHIERI, 2013). 


\subsection{Qualidade de vida}

Qualidade de vida se trata da "percepção do indivíduo de sua inserção na vida, no contexto da cultura e sistemas de valores nos quais ele vive e em relação aos seus objetivos, expectativas, padrões e preocupações" (CARVALHO,2019, p.oI).

Acredita-se com isso, que qualidade de vida, envolve: bem-estar: físico, mental, espiritual, psicológico e emocional de cada indivíduo (FERRO, 2013).

Ter boas relações entre amigos, familiares, no trabalho, bem como ter acesso à saúde, educação, habitação, saneamento básico, entre diversos outros fatores, contribui para que cada indivíduo alcance verdadeiramente a sua qualidade de vida (MARQUES, 2020, p.or).

\subsection{Qualidade de Vida na Doença de Alzheimer}

Quando se trata da qualidade de vida em pacientes com alzheimer, não se deve dar importância apenas ao tratamento com medicamentos, mas também, aos exercícios físicos, bem como oferecer qualidade de vida ao paciente pensando na parte motora, para que não ocorram quedas, que podem levar o paciente a situações mais graves (CASILLAS, 2018 e CAVALCANTI, 2019), uma alimentação correta e aos cuidados pessoais, usar estratégias compensatórias de adaptação para estimular o paciente no que tange as funções cognitivas comprometidas, na realização de atividades diárias, conhecida como, estimulação cognitiva ou reabilitação cognitiva, e também um trabalho motor, sendo um papel do fisioterapeuta neste contexto (KOTTKE, 2012).

A estimulação cognitiva é uma outra solução terapêutica, não medicamentosa à doença de alzheimer sendo essencial não só para o treino das funções cerebrais perdidas como também, no controle de outros sintomas, promovendo assim, o bem-estar do paciente e o estimulando para uma maior qualidade de vida, mesmo com as limitações que a doença apresenta (BERNARDO, 2018).

Afirma-se que, não se pode curar a doença de alzheimer, mas enquanto existirem maneiras de amenizar sua progressão, ampliar e melhorar a qualidade enquanto ela ainda existir, deve-se proporcionar essa condição a todo paciente comprometido ou não pela doença (CARVALHO, 2019 e FERRO, 2013). 


\subsection{Fisioterapia Precoce no Alzheimer: Técnicas Utilizadas e seus Benefícios}

Ao se falar sobre a fisioterapia precoce em pacientes com alzheimer constata-se que, este trabalho é importante sendo realizado duas ou três vezes, por semana, em desde o início da doença e, que apresentam indícios de: dificuldade em andar ou equilibrar, por exemplo, ajudando a retardar o avançar da doença e oferecendo autonomia ao doente, por um maior período de tempo (LINS, 2019). "A intervenção fisioterapêutica pode contribuir em qualquer fase da doença de Alzheimer para manter o indivíduo mais ativo e mais independente possível, seja no domicílio, seja numa instituição" (KOTTKE, 2012, p.89).

$\mathrm{Na}$ fase inicial da doença quando o doente ainda caminha, pode-se fazer uma intervenção fisioterapêutica ampliando a força nos músculos, sendo a psicomotricidade estimulada, com maior ênfase à lateralidade, à auto imagem, à percepção corporal, coordenação e ao equilíbrio (BASTOS, 2016).

Assim, também, caso o paciente apresente mudanças em sua postura, o fisioterapeuta trabalhará com ele exercícios de alongamentos de grupos musculares encurtados; se for detectada alteração no equilíbrio, trabalha-se com exercícios que recuperem para esta condição (KAUFFMAN, 2019)

Com tudo isso, devem ser incorporados no tratamento do paciente com Alzheimer: exercícios de alongamento e mobilidade corporal, visando aumentar o comprimento de tecidos moles a fim de melhorar a flexibilidade da articulação e a mobilidade, melhorando a rigidez e a dor, já que a mobilização mantém a amplitude de movimento, o que facilita a realização de atividades de vida diária e transferências. (ABBAS, 2012).

Também é importante que sejam "evitadas as atrofias por desuso e fraqueza muscular, os encurtamentos de tecidos moles e as deformidades esqueléticas" (REBELATTO et al., 2014, p.or).

Exercícios terapêuticos direcionados para os padrões do funcionamento cardiorrespiratório também são muito importantes, uma vez que no indivíduo portador da doença de Alzheimer a capacidade funcional da fala, a respiração, expansão torácica e função venosa vão diminuindo progressivamente (O'SULLIVAN et. al., 2018). 


\section{METODOLOGIA}

O trabalho foi realizado com pesquisa bibliográfica, computador, apostilas textos, atividades cotidianas, enfim tudo o que servir de apoio. Em seminários, simpósios, em todo e qualquer evento e fundamentalmente, nos referenciais teóricos bibliográficos, o texto e os contextos são destaques como força motriz, para conduzirem ao bom entendimento deste assunto de forma eficaz (FERNANDES, 2000).

Realiza-se, então, uma pesquisa bibliográfica, através da técnica de revisão de literatura, sendo utilizados artigos relacionados ao tema em questão, em língua inglesa e portuguesa, e consulta nas bases de dados PubMed-Medline da United States National Library of Medicine, National Institutes of Health e Scielo - Scientific Electronic Library Online e Google Acadêmico, separando os artigos de $201 \mathrm{I}$ até 202I. Sendo que os descritores/palavras-chave utilizados nesta pesquisa: fisioterapia neuro lógica, atuação do fisioterapeuta e doença de Alzheimer.

Para o desenvolvimento deste trabalho, foram realizadas pesquisas bibliográficas intentando construir um referencial teórico capaz de possibilitar o entendimento do tema, para que possam sanar as hipóteses já elencadas e as que surgirão através do estudo.

\section{CONCLUSÃO}

Ao se falar sobre a fisioterapia em paciente com alzheimer constata-se que, este trabalho é importante sendo realizado duas ou três vezes, por semana, em desde o início da doença e, que apresentam indícios de: dificuldade em andar ou equilibrar, por exemplo, ajudando a retardar o avançar da doença e oferecendo autonomia ao doente, por um maior período de tempo.

Assim, existem medicações atualmente, que estabilizam a doença ou diminuem a velocidade de perda funcional em cerca de cinco anos ou mais, sendo o caso da memantina, que foi descoberta pelo Alois Alzheimer, podendo oferecer mais tempo com qualidade de vida, ao paciente e aos familiares.

Depreende-se que, apesar de a doença não ter cura, as medicações, desde que bem administradas, junto com um trabalho fisioterapêutico bem feito, oferecem: conforto, 
alívio e qualidade de vida para o doente. Assim, não se cuida da doença, e sim da pessoa humana que a possui, bem como, seus familiares e cuidadores.

\section{REFERÊNCIAS}

ABBAS AK, Lichtman AH, Pillai S. Imunologia celular e molecular. 7 ed. Rio de Janeiro: Elsevier 545, 2012.

ALCHIERI JC, Cruz RM. Avaliação Psicológica: conceito, métodos e instrumentos. São Paulo: Casa do Psicólogo. 128, 2013.

BAllone, G. J. Doença de Alzheimer. In PsiqWeb Psiquiatria Geral. Atualizado em 2012. Disponível em: https://pesquisa.bvsalud.org/portal/resource/pt/lis-LISBRI.I-I7769. Acesso em: i8 de junho de 2021.

BAPTISTA A. Perturbações do medo e da ansiedade. Uma perspectiva evolutiva e desenvolvimental. In I. Soares (Ed.), Psicopatologia do desenvolvimento. Trajectórias (in)adaptativas ao longo da vida. Coimbra: Quarteto. 20Ir.

BASTOS, C. C.; GUIMARÃES, L. S. e SANTOS, M. L. A. S. 2oi6. Mal de Alzheimer: $\begin{array}{lllll}\text { uma } & \text { Visão } & \text { Fisioterapêutica. } & \text { Disponível }\end{array}$ www.nead.unama.br/bibliotecavirtual/revista/lato/pdf/lato4rar7.pdf \#seach=\%22mal\%2 ode\%2oDA\%3A\%\%2ouma\%2ovis\%C3\%A30\%2ofisioterap\%C3\%AAutica\%22. Acesso em: I8 de junho de 2021.

BAUER ME. Estresse. Como ele abala as defesas do corpo. Ciência Hoje. Instituto de Pesquisas Biomédicas e Faculdade de Biociências, Pontifícia Universidade Católica do Rio Grande do Sul. 30: 179, 2012.

BERNARDO, L. D. Revisão integrativa sobre o engajamento em ocupações de idosos com Alzheimer. Rev. Interinst. Bras. Ter. Ocup. Rio de Janeiro. v.I,n.3. p.386 - 407. 2017. 
BERNARDO, L. D. Idosos com Doença de Alzheimer: uma revisão sistemática sobre a intervenção da Terapia Ocupacional nas alterações em habilidades de desempenho. Cad. Bras. Ter. Ocup., São Carlos, v.26, n.4, p.926 - 942. 2018.

BERNARDO, L. D.; RAYMUNDO, T. M. Ambiente físico e social no processo de intervenção terapêutico ocupacional para idosos com Doença de Alzheimer e seus cuidadores: uma revisão sistemática da literatura. Cad. Bras. Ter. Ocup., São Carlos, v.6, n.2, p.463-477. 2018.

CARVALHO, Paulo. Geriatria: fundamentos, Clínica e Terapêutica. São Paulo: Editora Atheneu, 2019.

CASILLAS J. Custo energético da marcha. In: Viel E, editor. A marcha humana, a corrida e o salto. São Paulo: Manole; 2018. p. I41-55.

CAVALCANTI. José Luiz de Sá e Engelhardt. Eliasz. Aspectos da fisiopatologia da doença de Alzheimer esporádica. Revista Brasileira de Neurologia. Volume 48 № 4 out nov - dez, 20I2. Disponível em: http://files.bvs.br/upload/S/oroI8469/2012/v48n4/a3349.pdf. Acesso em: in de novembro de 2021.

CHARONE Cynthia. Tudo o que você precisa saber sobre doença de alzheimer. 202I. Disponível em: https://blog.cynthiacharone.com/doenca-de-alzheimer/. Acesso em: II de novembro de 202I.

CORREAA, S. E. S.; SILVA, D. B. Abordagem cognitiva na intervenção terapêutica ocupacional com indivíduos com a doença de Alzheimer. Rev. Bras. Gegiatr. Gerontol., v.12, n.3. p.463- 474. 2012.

CAOVIllA, Vera Pedrosa. A Doença de Alzheimer e o impacto na família. Revista a Terceira Idade. SESC, São Paulo, n. 22, p. 37-45, 2011. 
CUNHA, F. C. M., CUNHA, L. C. M., SILVA, H. M., COUTO, E. A. B. Abordagem funcional e centrada no cliente na reabilitação de idoso com demência de alzheimer avançada - relato de caso. Rev. Ter. Ocup. Univ. São Paulo, v. 22, n. 2, p.145-152. 2011.

FERNANDES, José. Técnicas de Estudo e Pesquisa. 2. ed. Goiânia: Kelps, 2000.

FERRO, A. O.; LINS, A. E. S.; FILHO, E. M. T. Comprometimento cognitivo e funcional em pacientes acometidos de acidente vascular encefálico: importância da avaliação cognitiva para intervenção na Terapia Ocupacional. Cad. Ter. Ocup. UFSCar, São Carlos, v.21, n.3, p. 521 - 527. 2013.

FLECK, Paula. Fundamentos do Treinamento de Força Muscular. Porto Alegre: Artmed, 2010.

FREITAS, E. V. et al. Tratado de geriatria e gerontologia. Rio de Janeiro: Guanabara Koogan, 2012.

GIL, A. C. Como elaborar projetos de pesquisa. 3. ed. São Paulo: Atlas, I994.

GORZONI ML, Pires SL. Aspectos clínicos da demência senil em instituições asilares. Rev Psiq Clín. 2016;33(I):18-23.

KAUFFMAN, Timothy L. Manual de reabilitação geriátrica. Rio de Janeiro: Editora Guanabara Koogan, 2019.

KOTTKE, F. J.; LEHMANN, J. F. Tratado de medicina e reabilitação de Krusen. 4. ed. São Paulo: Manole, 2012. v. 2.

LAKATOS, E. M.; MARCONI, M. A.: Fundamentos de Metodologia Científica. São Paulo: Ática, I999. 
LINS, V. S.; GOMES, M. Q. C. Terapia Ocupacional no cuidado ao idoso com demência: Uma revisão integrativa. Rev. Interinst. Bras. Ter. Ocup. Rio de Janeiro. v.3, n.I. p.II7 - I32. 2019 .

MACHADO, João Carlos Barbosa. Doença de Alzheimer. In: FREITAS, E. V. de et al, Tratado de Geriatria e Gerontologia, Rio de Janeiro: Guanabara Koogan S. A., 2012. p. 132I47.

O’SULLIVAN, S.; SCHMITZ, T. Fisioterapia: avaliação e tratamento. 4. ed. São Paulo: Manole, 2018.

PEÇANHA, M.A.P; NERI, V.C. Estudo Neuropatológico e Funcional da Doença de Alzheimer. Revista Científica da Faculdade de Medicina de Campos. v. 2, n.I. 2019.

REBELATTO, J. R. e MORELLI J. G. S. Fisioterapia geriátrica - a prática da assistência ao idoso. São Paulo, Manole, 2014.

XIMENES, M.A.; RICO, B.L.D.; PEDREIRA, R.Q. Doença de Alzheimer: a dependência e o cuidado. Revista Kairós Gerontologia, São Paulo. v.r7, n.2. 2014. 